\title{
Non-Line-of-Sight Around the Corner Human Presence Detection Using Commodity WiFi Devices
}

\author{
Shiwei Fang, Ron Alterovitz and Shahriar Nirjon \\ Department of Computer Science \\ University of North Carolina at Chapel Hill \\ \{shiwei,ron,nirjon\}@cs.unc.edu
}

\begin{abstract}
As robots penetrate into real-world environments, practical humanrobot co-existence issues such as the requirement for safe humanrobot interaction are becoming increasingly important. In almost every vision-capable mobile robot, the field of view of the robot is occluded by the presence of obstacles such as indoor walls, furniture, and humans. Such occlusions force the robots to be stationary or to move slowly so that they can avoid collisions and violations of entry into the personal spaces of humans. We see this as a barrier to robots being able to optimally plan motions with reasonable speeds. In order to solve this problem, we propose to augment the sensing capability of a robot by using a commodity WiFi receiver. Using our proposed method, a robot can observe the changes in the properties of received signals, and thus be able to infer whether a human is present behind the wall or obstacles, which enhances its ability to plan and navigate efficiently and intelligently.
\end{abstract}

\section{CCS CONCEPTS}

- Computing methodologies $\rightarrow$ Feature selection; • Computer systems organization $\rightarrow$ Sensors and actuators

\section{KEYWORDS}

WiFi, CSI, Robotics, Non-Line-of-Sight, Human Sensing

\section{ACM Reference Format:}

Shiwei Fang, Ron Alterovitz and Shahriar Nirjon. 2019. Non-Line-of-Sight Around the Corner Human Presence Detection Using Commodity WiFi Devices. In The 1st ACM International Workshop on Device-Free Human Sensing (DFHS'19), November 10, 2019, New York, NY, USA. ACM, New York, NY, USA, 5 pages. https://doi.org/10.1145/3360773.3360879

\section{INTRODUCTION}

With the increased deployment of robots in real-world scenarios, practical human-robot interaction issues such as safety and sociability are becoming increasingly important. With recent advances in robotic planning and control, although the reaction of robots is becoming more and more agile, for many robotics applications it is still not on par with the dynamics of humans. Given the limited capability of fast reaction, the demand for better sensing arises.

Permission to make digital or hard copies of all or part of this work for personal or classroom use is granted without fee provided that copies are not made or distributed for profit or commercial advantage and that copies bear this notice and the full citation on the first page. Copyrights for components of this work owned by others than ACM must be honored. Abstracting with credit is permitted. To copy otherwise, or republish, to post on servers or to redistribute to lists, requires prior specific permission and/or a fee. Request permissions from permissions@acm.org.

DFHS'19, November 10, 2019, New York, NY, USA

(C) 2019 Association for Computing Machinery.

ACM ISBN 978-1-4503-7007-3/19/11 . \$ \$15.00

https://doi.org/10.1145/3360773.3360879

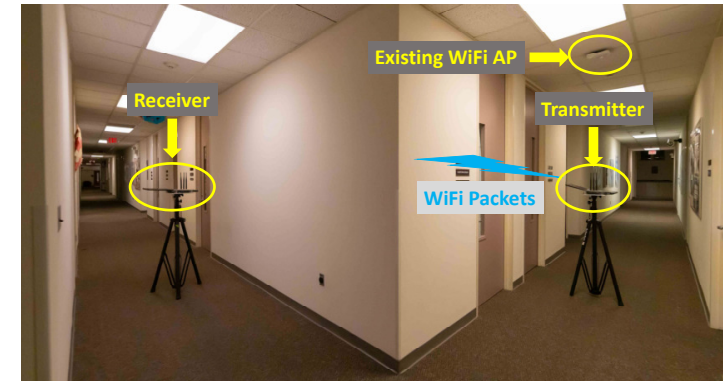

Figure 1: Our system consisting two WiFi devices, a transmitter which can be exiting WiFi Access Point (AP) and a receiver which can be WiFi card on the robot. The receiver receives packets transmitted by the transmitter then analyze the properties of the received packet to estimate whether human is present or not.

We hypothesize that - for a robot equipped with better sensing capabilities, its required reaction time can be relaxed, and safety and sociability can be increased.

Most on-board sensors of a robot, such as cameras, LIDAR, infrared, and ultrasonic sensors, require line-of-sight between the sensor and the target. These sensors may serve perfectly in the seen world, but in a crowded environment or places that have many blind spots, the line-of-sight requirement of these sensors makes it challenging for a robot to navigate safely and sociably. If the sensors were capable of $X$-ray vision for non-line-of-sight sensing, a robot could overcome these blind spots, plan its actions better, and ensure safer and more natural human-robot interaction for social robots.

Existing works have studied the seeing around the corner problem in robotics using vision [14, 4] and acoustics [3]. Cameras used in these systems are expensive, special, and require adequate lighting in the environment. Sound of footsteps is used to localize humans [3], but these solutions are not effective unless the environment is quiet and humans are walking as opposed to just standing around the corner. Recent works have exploited radio frequency (RF) signal's capability to penetrate walls to detect human figures $[1,19,18]$, but these solutions require large antenna arrays and moving human figures.

In this paper, we study the problem of determining whether a human is present around the corner in non-line-of-sight situations by using only commodity WiFi devices. We propose a solution that relies upon commodity WiFi devices and extracts features from received WiFi signals. In addition to standard coarse-grained signal power measurements such as Received Signal Strength Indicator (RSSI), we perform multipath estimation using fine-grained Channel State Information (CSI). We also extract features that represent 


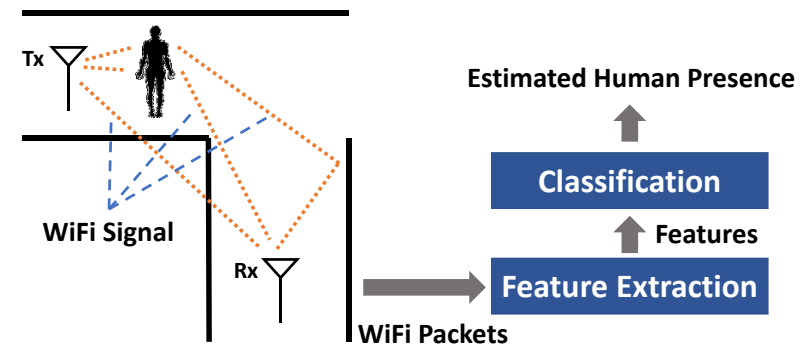

Figure 2: System Overview. The transmitter sends WiFi packets where the signal can travel directly, pass through the human body, and reflected to the receiver. The receiver receives the packet and calculates the properties of the signal. Then our system extracts the features which are later used for classification on human presence. signal variability over time. Utilizing all these features, we model and classify the state of the environment.

Our solution works in real-time and is not dependent on the environments. Unlike prior works, we require only $50 \mathrm{~ms}$ for sensing, which makes it suitable for indoor mobile robot applications. Since we rely on commodity WiFi devices, the solution can be easily incorporated into mobile robots. The extended sensing capability does not add significant cost as many robots already use WiFi for connectivity. We evaluate our solution in multiple unseen environments (i.e., training and testing environments are different) to demonstrate the robustness of our system. We compare our solution with existing ones and show that in challenging environments, the proposed solution outperforms the existing ones.

\section{SYSTEM OVERVIEW}

We study the problem of detecting human presence in aroundthe-corner situations using commodity WiFi devices. An example scenario is depicted in Figure 1. A WiFi transmitter, such as a WiFi $\mathrm{AP}$ in a building, and a WiFi receiver, such as a WiFi device mounted on a robot, are placed on an L-shaped corridor in a non-line-of-sight setup. The goal of the receiver is to determine whether there are humans on the other side where the WiFi transmitter is.

An overview of how the proposed solution is shown in Figure 2. Tx and Rx refer to the transmitter and the receiver, respectively. When the transmitter sends a packet, the WiFi signal is broadcasted in all directions. The signal travels through the walls and reaches the receiver on the shortest path. Some of the signals get attenuated by the human body (if present) before reaching the receiver. ${ }^{1}$ Signals also get reflected multiple times before the receiver receives them. Hence, the received signal is a combination of all these signals traveling on different paths. A number of consecutive WiFi packets are retrieved, a set of features is computed, and classified to determine if a human is present around the corner.

\section{WIFI FEATURE EXTRACTION}

This section describes the WiFi features that the system uses to represent the RF environment. All features are extracted from the same number of received packets.

\footnotetext{
${ }^{1}$ The human is occluded by the corner, which results in no direct line-of-sight (LOS) path for the WiFi signal to pass through the human body and then directly received by the receiver. All such signals are attenuated further by the walls and other objects in the environment.
}

\subsection{Received Signal Strength Features}

The Received Signal Strength Indicator (RSSI) is an estimate of power in the received signal at an RF client. The signal strength is affected by multiple factors such as the distance between Tx and Rx, obstacles, Tx strength, and Rx antenna's property. Since the human body attenuates WiFi signals, e.g., by absorbing signals, it changes the RSSI value. Hence, we use RSSI as one of the features to represent the RF environment.

For each packet $P$, the WiFi card reports three RSSI values: $R S S I_{a}$, $R S S I_{b}$, and $R S S I_{c}$, where each value corresponds to one of the three receiving antennas. We calculate the mean and the variance of each RSSI from the $N$ received packets: $(x \in\{a, b, c\})$

$$
\mu_{R S S I_{x}}=\frac{1}{N} \sum_{i=1}^{N} R S S I_{x_{i}}, \sigma_{R S S I_{x}}^{2}=\frac{1}{N} \sum_{i=1}^{N}\left(R S S I_{x_{i}}-\mu_{R S S I_{x}}\right)
$$

\subsection{Effective Signal to Noise Ratio Features}

The Effective Signal Noise Ratio (SNR), which measures the quality of the WiFi signals, is affected by environmental changes. We calculate the effective SNR for four modulation schemes, i.e., BPSK, QPSK, 16QAM, and 64QAM. The effective SNR is calculated from the channel state information (CSI), which is described next. Since we only use one transmission antenna, it is a Single Input Multiple Output (SIMO) system. The calculated effective SNR is a $1 \times 4$ vector $\left(S N R_{0}, S N R_{1}, S N R_{2}, S N R_{3}\right)$, where each element corresponds to a modulation scheme.

Similar to RSSI, we calculate the mean and the variance for each effective SNR across the $N$ received packets, which become parts of the feature vector: $(j=0,1,2,3)$

$$
\mu_{S N R_{j}}=\frac{1}{N} \sum_{i=0}^{N} S N R_{j_{i}} \quad, \quad \sigma_{S N R_{j}}^{2}=\frac{1}{N} \sum_{i=1}^{N}\left(S N R_{j_{i}}-\mu_{S N R_{j}}\right)
$$

\subsection{Signal Tendency Index}

The Signal tendency index (STI) $[20,21]$ is based on Procrustes analysis to compare shape similarity across different packets. It is calculated from the Channel State Information (CSI) containing finegrained information of both the magnitude and the phase of each subcarrier between each transmitter-receiver antenna pair [17].

For the CSI vector $\left(H_{1}^{t}, H_{2}^{t}, \ldots, H_{n}^{t}\right)$, at time step, $t=1,2, \ldots, N$, we standardize the values by subtracting the mean and dividing by the standard deviation:

$$
\begin{gathered}
\hat{H}^{t}=\frac{\left[H_{1}^{t}-\bar{H}^{t}, H_{2}^{t}-\bar{H}^{t}, \ldots, H_{n}^{t}-\bar{H}^{t}\right]}{\sigma\left(H^{t}\right)} \\
\bar{H}_{1}^{t}=\frac{1}{n} \sum_{i=0}^{n} H_{i}^{t} \quad, \quad \sigma\left(H^{t}\right)=\sqrt{\frac{\sum_{i=0}^{t}\left(H_{i}^{t}-\bar{H}^{t}\right)^{2}}{n}}
\end{gathered}
$$

The STI between two consecutive packets is $S=\left\|\hat{H}^{t}-H^{\hat{t}-1}\right\|$, which is the Euclidean distance between the curves in STI metrics and larger STI value means greater difference. An example of STI values between different packets is shown in Figure 3. The changes between packets $20-40$ are larger than the rest as they are collected while a person walks, which severely distorts the WiFi signal between packets. As for the empty space (0-20) and a person standing still (40-60), the difference is similar to the environment is almost static. We also observe that even in the empty space, CSI is not stable. 


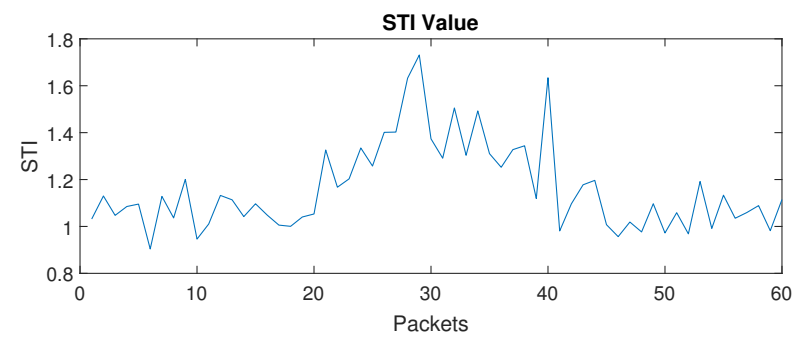

Figure 3: Example of STI value across different packets. Packets 020: empty environment. Packets 20-40: a person is walking. Packets 40-60: a person is standing still.

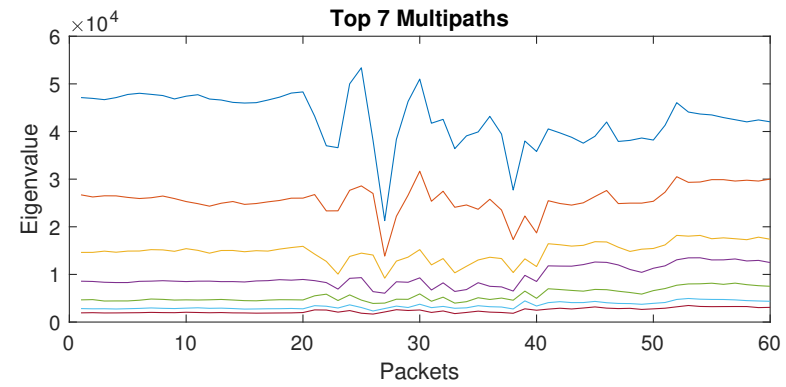

Figure 4: Example of eigenvalues for multipath analysis in different packets. Packet 0-20: empty environment. Packet 20-40: a person is walking. Packet 40-60: a person is standing still.

With $N$ packets received, we calculate $N-1$ STI values. The mean and variance of the STI are then computed and used as a feature.

\subsection{Multipath}

As the human body attenuates WiFi signals, the multipath profile of the WiFi environment changes. When we have many reflectors in the environment (e.g., a crowded scene), the number of multipath is large, but the difference in energy of different paths is small. For a relatively less crowded scene, the number of multipath is less, but the energy differences are high. This property of multipath can be modeled from the WiFi CSI values.

The CSI measurement, $X$, which contains both magnitude and phase information, can be used to compute the angle of arrival (AoA) [15] of a signal. For example, in recent WiFi-based localization algorithms [11, 5], the AoA of the direct path (which is relevant to the localization problem) is isolated by taking the eigenvector of the matrix, $X X^{H}$, for which, the eigenvalue is zero. The eigenvector goes through further processing to obtain the direct path.

Inspired by this, one can compute the AoA corresponding to each eigenvalue to create a multipath profile of the WiFi environment. However, we propose that, in order to model the multipath profile, we do not necessarily have to compute the AoAs. Instead, we can take the top $k$ largest eigenvalues of $X X^{H}$ to have the simplest way to create a feature that inherently represents the multipath profile. An example of the proposed multipath-based features is shown in Figure 4 where we use top seven eigenvalues. We observe that the three behind-the-wall cases: empty space, a person walking, and a person standing is clearly distinguishable by the proposed feature.
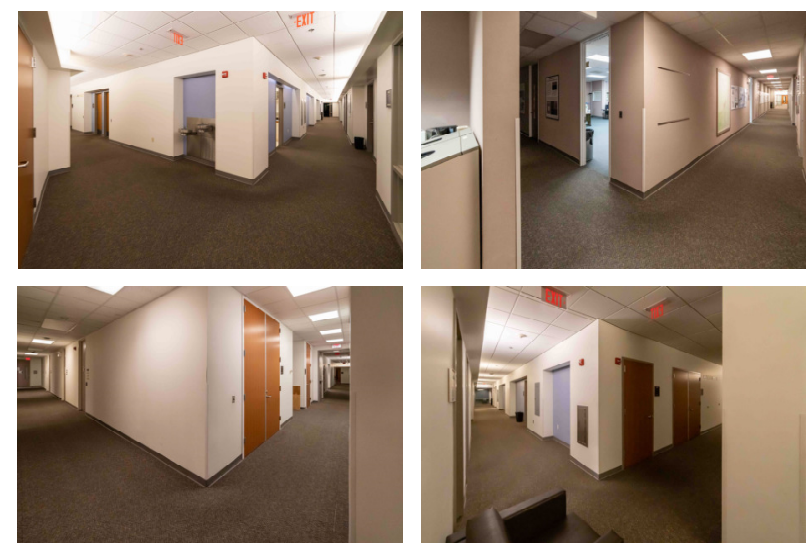

Figure 5: Data collection Environments. Each corner is different from one another, the layout and materials around each corner creates totally different WiFi propagation characteristics.

\section{HUMAN PRESENCE DETECTION}

We formulate the non-line-of-sight around the corner human detection as a classification problem having three classes: empty, standing, and moving. We implement a Random Forest [12] classifier that uses the feature vector described in the previous section. We empirically determine that extracting features from a total of $N=50$ packets result in the best classification accuracy while keeping the sensing delay as low as $50 \mathrm{~ms}$.

\section{IMPLEMENTATION}

We implement the proposed system using two laptops having Intel 5300 WiFi Network Interface Cards (NICs). We use Linux CSI tool [8] for collecting PHY layer CSI information from transmitted packets. We operate in the $5 \mathrm{GHz}$ WiFi spectrum to avoid firmware limitations [7]. The transmitter operates in the injection mode and the receiver operates in the monitor mode.

To train and test the classifier, we collect WiFi data from seven different corners across different floors of a four-storied building by placing the transmitter and the receiver at varying locations. Some example setups are shown in Figure 5. The laptop used as AP transmits WiFi packets over one antenna $\left(N_{T X}=1\right)$ and the receiver receives on three antennas $\left(N_{R X}=3\right)$. The packets are transmitted at $1000 \mathrm{~Hz}$, which results in a sensing delay of $50 \mathrm{~ms}$ for 50 packets used for feature extraction. Each received packet contains the RSSI and CSI for each antenna. The CSI is a $N_{T x} \times$ $N_{R x} \times 30$ matrix where 30 is the number of subcarriers in the WiFi channel reported by the Linux CSI tool.

We consider three scenarios: 1) no one is in the environment, 2) a person is standing around the corner who is occluded by the wall from the receiver's viewpoint, and 3) a person walking in the occluded area.

\section{RESULTS}

To evaluate and examine the robustness of our system, the training and testing datasets are collected from different floors. Hence, our model has not seen any example from the testing environments. We report the precision, recall, and F1 score for each class, and the overall accuracy in Table 1. 


\begin{tabular}{|l|c|c|c|c|}
\hline Class & Precision & Recall & F1 & Overall Accuracy \\
\hline Empty & 0.48 & 0.59 & 0.53 & \\
\cline { 1 - 3 } Standing & 0.47 & 0.36 & 0.4 & \multirow{2}{*}{$61.37 \%$} \\
\cline { 1 - 3 } Moving & 0.89 & 0.89 & 0.89 & \\
\hline
\end{tabular}

Table 1: Classification results with three classes.

We also show the result of binary classification: empty and occupied by combining the standing and moving classes into a single class. The result is shown in Table 2.

\begin{tabular}{|l|c|c|c|c|}
\hline Class & Precision & Recall & F1 & Overall Accuracy \\
\hline Empty & 0.5 & 0.33 & 0.4 & \multirow{2}{*}{$66.47 \%$} \\
\hline Occupied & 0.71 & 0.83 & 0.77 & \\
\hline
\end{tabular}

Table 2: Classification results with two classes.

\section{DISCUSSION}

At first glance, the results reported in this paper may seem to have fallen short of what similar systems have reported $[21,16]$. However, there is a major difference in how the evaluation is done and how the experiment ( $\mathrm{Tx}$ and $\mathrm{Rx}$ ) was setup.

We conduct experiments to demonstrate the expected performance of the proposed system in real-world scenarios that have a completely new environment. In other words, we train and test on completely different environments. On the other hand, existing works [21, 16] collect a single dataset and then split it into training and testing sets. Due to the limited number of locations, their training and testing sets contain the same transmitter and receiver location pairs-which makes the classification task easier. If we split our dataset into training and testing, and redo the experiment, we achieve results similar to [16], which is reported in Table 3. We also perform binary classification, i.e., empty vs. occupied, by splitting the dataset. The result is shown in Table 4.

Furthermore, the experimental setup in existing works considers line-of-sight situations where the human is not occluded. These systems use 1500 packets at $50 \mathrm{~Hz}$, which results in 30 s sensing time, which is $600 \mathrm{X}$ slower than our solution.

The difference in the two evaluation methods revealed that if our system is deployed to a specific area, it can continuously collect more data for training and can potentially see a significant performance boost. Thus, the results reported in this paper should be treated as the lower bound if the system is deployed to a completely new environment, which can still provide important information to improve safety.

\begin{tabular}{|l|c|c|c|c|}
\hline Class & Precision & Recall & f1 & Overall Accuracy \\
\hline Empty & 0.95 & 0.95 & 0.95 & \multirow{2}{*}{$94.49 \%$} \\
\cline { 1 - 3 } Standing & 0.96 & 0.94 & 0.95 & \\
\hline Moving & 0.92 & 0.94 & 0.93 & \\
\hline
\end{tabular}

Table 3: Classification results with 3 classes and evaluated through splitting training and testing data set.

\begin{tabular}{|l|c|c|c|c|}
\hline Class & Precision & Recall & f1 & Overall Accuracy \\
\hline Empty & 1 & 0.97 & 0.98 & \multirow{2}{*}{$98.85 \%$} \\
\hline Occupied & 0.98 & 1 & 0.99 & \\
\hline
\end{tabular}

Table 4: Classification results with 2 classes and evaluated through splitting training and testing data set.

\section{RELATED WORK}

Prior works have used WiFi to track humans through walls [2] but these solutions require the human subjects to be continuously moving. WiFi has also been used for occupancy detection $[21,16]$ and crowd counting [22], but these systems require the subject to cut through the direct line between the transmitter and the receiver - which does not apply to around the corner situations. While WiFi has been used for imaging [9], these solutions require special antennas and antenna arrays which are not practical for indoor robots due to space and weight requirements. WiFi has also been used in 3D imaging $[10,6]$, but these solutions require a pair of transmitter and receiver to travel in a specific pattern.

Frequency modulated Continuous Wave (FMCW) technique has been explored to capture human figures through the wall $[1,19,18]$. While FMCW signal provides better granularity, these systems require custom devices to operate at lower frequencies for a better penetration capability which makes the antenna size large. Throughwall radars such as [13] are unsuited for our application due to size and complexity of the system.

\section{CONCLUSION AND FUTURE WORK}

We present a system that detects human presence in non-line-ofsight around-the-corner situations using only commodity WiFi devices. We evaluate our system in different unseen environments to demonstrate its robustness. In our future work, we plan to explore the possibility of extracting more information from CSI measurements by analyzing multipath profile, which can be combined with the $3 \mathrm{D}$ model of the environment to better estimate the effects of the human body on the WiFi signal. We also plan to collect more data and conduct experiments to observe whether deep learning models can increase performance.

\section{ACKNOWLEDGMENT}

This paper was supported, in part, by NSF grants CNS-1816213 and CNS-1704469.

\section{REFERENCES}

[1] Fadel Adib, Chen-Yu Hsu, Hongzi Mao, Dina Katabi, and Frédo Durand. 2015. Capturing the human figure through a wall. ACM Transactions on Graphics (TOG) 34, 6 (2015), 219.

[2] Fadel Adib and Dina Katabi. 2013. See through walls with WiFi! Vol. 43. ACM.

[3] Inkyu An, Myungbae Son, Dinesh Manocha, and Sung-eui Yoon. 2018. Reflection-Aware Sound Source Localization. In 2018 IEEE International Conference on Robotics and Automation (ICRA). IEEE, 66-73.

[4] Bin Bai, Yuchen He, Jianbin Liu, Yu Zhou, Huaibin Zheng, Songlin Zhang, and Zhuo Xu. 2017. Imaging around corners with single-pixel detector by computational ghost imaging. Optik 147 (2017), 136-142.

[5] Nicolai Czink, Markus Herdin, Hüseyin Özcelik, and Ernst Bonek. 2004. Number of multipath clusters in indoor MIMO propagation environments. Electronics letters 40, 23 (2004), 1498-1499.

[6] Saandeep Depatla, Lucas Buckland, and Yasamin Mostofi. 2015. $\mathrm{X}$-ray vision with only wifi power measurements using rytov 
wave models. IEEE Transactions on Vehicular Technology 64, 4 (2015), 1376-1387.

[7] Jon Gjengset, Jie Xiong, Graeme McPhillips, and Kyle Jamieson. 2014. Phaser: Enabling phased array signal processing on commodity WiFi access points. In Proceedings of the 20th annual international conference on Mobile computing and networking. ACM, 153-164.

[8] Daniel Halperin, Wenjun Hu, Anmol Sheth, and David Wetherall. 2011. Tool release: Gathering $802.11 \mathrm{n}$ traces with channel state information. ACM SIGCOMM Computer Communication Review 41, 1 (2011), 53-53.

[9] Donny Huang, Rajalakshmi Nandakumar, and Shyamnath Gollakota. 2014. Feasibility and limits of wi-fi imaging. In Proceedings of the 12th ACM Conference on Embedded Network Sensor Systems. ACM, 266-279.

[10] Chitra R Karanam and Yasamin Mostofi. 2017. 3D throughwall imaging with unmanned aerial vehicles using WiFi. In 2017 16th ACM/IEEE International Conference on Information Processing in Sensor Networks (IPSN). IEEE, 131-142.

[11] Manikanta Kotaru, Kiran Joshi, Dinesh Bharadia, and Sachin Katti. 2015. Spotfi: Decimeter level localization using wifi. In ACM SIGCOMM Computer Communication Review, Vol. 45. ACM, 269-282.

[12] Andy Liaw, Matthew Wiener, et al. 2002. Classification and regression by randomForest. $R$ news 2,3 (2002), 18-22.

[13] John Peabody Jr, Gregory L Charvat, Justin Goodwin, and Martin Tobias. 2012. Through-wall imaging radar. Technical Report. Massachusetts Institute of Technology-Lincoln Laboratory Lexington United States.

[14] Charles Saunders, John Murray-Bruce, and Vivek K Goyal. 2019. Computational periscopy with an ordinary digital camera. Nature 565, 7740 (2019), 472.
[15] Ralph Schmidt. 1986. Multiple emitter location and signal parameter estimation. IEEE transactions on antennas and propagation 34, 3 (1986), 276-280.

[16] Chenshu Wu, Zheng Yang, Zimu Zhou, Xuefeng Liu, Yunhao Liu, and Jiannong Cao. 2015. Non-invasive detection of moving and stationary human with wifi. IEEE fournal on Selected Areas in Communications 33, 11 (2015), 2329-2342.

[17] Zheng Yang, Zimu Zhou, and Yunhao Liu. 2013. From RSSI to CSI: Indoor localization via channel response. ACM Computing Surveys (CSUR) 46, 2 (2013), 25.

[18] Mingmin Zhao, Tianhong Li, Mohammad Abu Alsheikh, Yonglong Tian, Hang Zhao, Antonio Torralba, and Dina Katabi. 2018. Through-wall human pose estimation using radio signals. In Proceedings of the IEEE Conference on Computer Vision and Pattern Recognition. 7356-7365.

[19] Mingmin Zhao, Yonglong Tian, Hang Zhao, Mohammad Abu Alsheikh, Tianhong Li, Rumen Hristov, Zachary Kabelac, Dina Katabi, and Antonio Torralba. 2018. RF-based 3D skeletons. In Proceedings of the 2018 Conference of the ACM Special Interest Group on Data Communication. ACM, 267-281.

[20] Han Zou, Baoqi Huang, Xiaoxuan Lu, Hao Jiang, and Lihua Xie. 2016. A robust indoor positioning system based on the procrustes analysis and weighted extreme learning machine. IEEE Transactions on Wireless Communications 15, 2 (2016), 1252-1266.

[21] Han Zou, Yuxun Zhou, Jianfei Yang, Weixi Gu, Lihua Xie, and Costas Spanos. 2017. Freedetector: Device-free occupancy detection with commodity wifi. In 2017 IEEE International Conference on Sensing, Communication and Networking (SECON Workshops). IEEE, 1-5.

[22] Han Zou, Yuxun Zhou, Jianfei Yang, and Costas J Spanos. 2018. Device-free occupancy detection and crowd counting in smart buildings with WiFi-enabled IoT. Energy and Buildings 174 (2018), 309-322. 